\title{
Assessment of Hepatic Arterial Anatomy Prior to Organ Recovery
}

\author{
Keith J. Roberts ${ }^{1}$, Deep J. Malde ${ }^{1}$, Brook Adams ${ }^{1}$, James Hodson ${ }^{2}$, Maria Sheridan ${ }^{3}$, Ernest Hidalgo ${ }^{1}$ \\ ${ }^{1}$ Liver Transplant Unit, St James University Hospital, Leeds, UK \\ ${ }^{2}$ Medical Statistician, University Hospitals Birmingham, Birmingham, UK \\ ${ }^{3}$ Hepatobiliary Radiologist, St James University Hospital, Leeds, UK \\ Email: j.k.roberts@bham.ac.uk
}

Received September 20, 2012; revised October 27, 2012; accepted November 3, 2012

\begin{abstract}
Iatrogenic hepatic arterial injury during organ recovery increases ischaemic times and risk of hepatic artery thrombosis. A review of CT imaging prior to organ recovery would alert retrieving surgeons to the presence of anatomical variants. This study aimed to identify the proportion of donors with coincidental CT scans for review and the ability of organ retrieval surgeons to interpret these images. Consecutive organ donors with coincidental abdominal contrast enhanced CT scans were assessed by review of an electronic radiology database. These images, with additional cases, were blindly reviewed by organ recovery surgeons to assess their ability to define anatomy. 13/156 donors had coincidental imaging for review. Using 23 CT sequences, the median positive and negative predictive value of surgeons to correctly describe right hepatic arterial anatomy was 0.83 and 0.94 , of the left hepatic anatomy was 0.75 and 0.94 respectively. The availability of CT imaging for review prior to donation is low. However, when available, surgeons can correctly define hepatic arterial anatomy in the majority of cases. A practice of routinely reviewing available imaging prior to organ recovery would be expected to decrease iatrogenic arterial injury.
\end{abstract}

Keywords: Organ Donation; Arterial Injury; Prevention; Computerized Tomography

\section{Introduction}

Anatomical variations of the arterial supply of the liver are not uncommon. The normal "textbook" anatomy of the hepatic artery is observed in approximately three quarters of humans with replaced or accessory right and left hepatic arteries responsible for the vast majority of "abnormal" cases $[1,2]$. The technique of liver retrieval for transplantation aims to minimise injury to the donated organ occurring during procurement, storage and implantation. Injury can be in the form of ischaemia-reper-fusion or damage to the liver itself, hilar structures or inferior vena cava. These latter injuries typically occur during the process of organ retrieval. Iatrogenic arterial injury occurs during organ procurement necessitating reconstruction prolonging ischaemic duration, predisposes to hepatic artery thrombosis and increases the need for retransplantation $[3,4]$ and may, rarely, render an organ unusable [5].

Computerised tomographic (CT) imaging permits an assessment of vascular anatomy. If CT was performed upon organ donors prior to organ retrieval a preoperative assessment of the arterial anatomy could be performed. It is, however, unethical to submit organ donors to investigations unnecessary to their medical management prior to donation. Thus only those patients who have coincidental imaging could be reviewed prior to organ procurement.

The aim of this study was to assess how many donors have coincidental imaging of their abdomen and to further identify whether organ procurement surgeons can correctly identify hepatic arterial anatomy by interpretation of these scans.

\section{Methods}

This project is comprised of two components; firstly to identify how many donors have coincidental imaging, consecutive organ donors at the Leeds General Infirmary or St James Hospital Leeds were identified from a national database (personal communication with NHS Blood and Transplant, Bristol, United Kingdom). These patients' records were searched for CT imaging available for review. Patients were included if they had a CT sequence of the abdomen including views of the liver, coeliac axis and superior mesenteric artery origins. The time limit for inclusion in the study was August 2004 to November 2010. This start point was selected as this was the date when an electronic computer based radiology software package replaced printed x-ray film. 
Secondly, the ability of liver procurement surgeons to accurately describe hepatic arterial anatomy using CT scans was assessed. The surgeons active at St James Hospital at the end of the study period were asked to participate $(\mathrm{N}=7$; all senior surgical trainees or fellows able to independently perform abdominal multiorgan retrieval). To provide a realistic case load all donors identified from the above search were used for review. As the scans were coincidental there was no formal protocol for processing the images or providing arterial or portal venous phase contrast. Each scan was reviewed by a consultant hepatobiliary radiologist (MS) for this study. On review of these images, predictably, there was a low number of cases of replaced/accessory hepatic arteries (replaced/accessory right hepatic artery, rRHA, 1 of 13 and replaced/accessory left hepatic artery, rLHA, 3 of 13). Thus additional patients, undergoing liver or pancreatic resection at St James' hospital, were identified. Their arterial anatomy was defined at the time of the operation. To increase the proportion of subjects with replaced/ accessory hepatic arteries only every fourth patient with normal anatomy was included.

Ten additional CT scans were available for review. In total there were 23 patients comprising 13 with no replaced hepatic arteries; of the ten remaining cases there were 5 with rLHA and 6 with rRHA, 1 had both rLHA and rRHA.

The surgeons and radiologist were blinded to the actual arterial anatomy. For each of the surgeons the sensitivity, specificity, positive and negative predictive values (PPV + NPV) were calculated. Data is presented as median (interquartile range). Interobserver agreement between retrieval surgeons was assessed using Fleiss' Kappa on a scale of 0 to 1 . A medical statistician advised and provided the analyses $(\mathrm{JH})$.

Surgeons were also asked to describe other vascular anatomy that would be relevant to liver organ retrieval.

\section{Results}

\subsection{The Incidence of Coincidental Abdominal CT Imaging for Review amongst Organ Donors}

Over the 71 month study period there were 156 donors of which 13 (8\%) had coincidental CT imaging suitable for review. Patients suffering a traumatic death were the most likely group to have coincidental imaging $(\mathrm{N}=9 / 24$ (38\%) vs. medical cause of death $\mathrm{N}=4 / 132$ (3\%), $\mathrm{P}=$ $<0.001)$.

\subsection{The Ability of Organ Retrieval Surgeons to Identify Replaced/Accessory Hepatic Arterial Anatomy on Review of CT Imaging}

The positive predictive value (PPV) and negative predic- tive value (NPV) of surgeons to correctly identify the presence, or not, of a rRHA was $0.83(0.69-0.92)$ and 0.94 (0.91 - 0.94) respectively (median, IQR).

The PPV and NPV of surgeons to correctly identify the presence, or not, of a rLHA was 0.75 (0.63 - 0.78) and 0.94 (0.89 - 0.95) respectively. This data including sensitivity and specificity are expressed in Table $\mathbf{1}$.

There was substantial agreement between surgeons describing the RHA and fair agreement when describing the LHA anatomy (Kappa $=0.64$ and 0.40 respectively).

The images did demonstrate other aberrant anatomy in several cases. There were two cases where the RHA arose from the coeliac trunk but passed posterior to the main portal vein (Figure 1). In one case the RHA was divided during the organ procurement. These cases were correctly identified on review of the imaging by $3 / 7$ and $1 / 7$ surgeons. In one case the common hepatic artery arose directly from the aorta, this was identified by 5/7 surgeons. In a final patient large left upper quadrant varices were present, identified by $3 / 7$ surgeons.

Table 1. Ability of liver retrieval surgeons $(N=7)$ to correctly identify hepatic arterial anatomy by interpretation of CT imaging in 23 cases.

\begin{tabular}{cccccc}
\hline & $\mathrm{N}$ & Sensitivity & Specificity & $\mathrm{PPV}$ & $\mathrm{NPV}$ \\
\hline \multirow{2}{*}{ Normal } & 13 & 0.77 & 0.80 & 0.83 & 0.73 \\
& & $(0.69-0.85)$ & $(0.70-0.80)$ & $(0.76-0.85)$ & $(0.66-0.80)$ \\
rRHA & $6^{*}$ & 0.83 & 0.94 & 0.83 & 0.94 \\
& & $(0.75-0.83)$ & $(0.88-0.97)$ & $(0.69-0.92)$ & $(0.91-0.94)$ \\
rLHA & 5 & 0.80 & 0.94 & 0.75 & 0.94 \\
& & $(0.60-0.80)$ & $(0.89-0.94)$ & $(0.63-0.78)$ & $(0.89-0.95)$ \\
\hline
\end{tabular}

Median scores are presented (inter-quartile range). Normal-type 1, rRHAreplaced right hepatic artery (type 2), rLHA - replaced left hepatic artery (type 3), "1 subject had both rRHA and rLHA (type 4) as described by Hiatt [1]

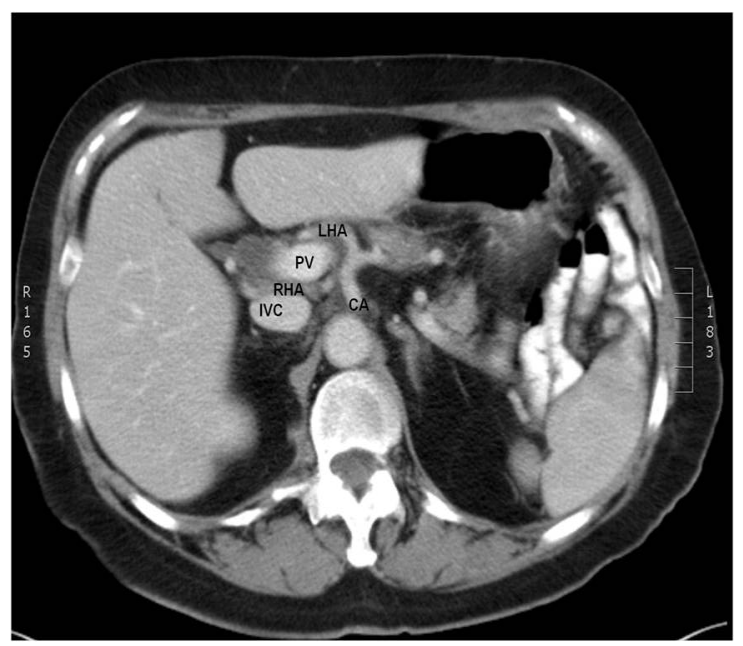

Figure 1. Right hepatic artery (RHA) arising from the coeliac axis (CA) but passing posterior to the portal vein (PV). In this case the RHA was divided requiring reconstruction. These images were not reviewed prior to organ retrieval. IVC - inferior vena cava, LHA —left hepatic artery. 


\section{Discussion}

This study aimed to identify how many organ donors have coincidental abdominal CT scans available at the time of organ procurement and how able retrieval surgeons are to interpret these images. The overall incidence of imaging was low at $8 \%$ though $38 \%$ of those dying following trauma had scans available for review. There were no cases of alternative methods of imaging the vascular anatomy, such as doppler ultrasound or angiography, identified in any of the donors. The other main finding was the high negative and positive predictive values associated with surgeons correctly describing hepatic arterial anatomy. The NPV was highest and is probably the most important observation. A lower PPV suggests that surgeons would be more likely to incurrectly suspect the presence of a replaced/accessory hepatic artery. Presumably the consequence of this would be increased care during the process of organ retrieval. Regardless of the availability of pre-operative imaging iatrogenic injury is avoidable with careful surgical technique and constant vigilance during the process of organ recovery.

The hepatic arterial anatomy, as considered normal by the classic textbook description, is observed in $76 \%$ of humans [1]. After studying 200 cadavers, Michels [6] proposed an internationally recognized classification of the hepatic artery variations into ten types, simplified into six types in 1994 [1]. In liver transplantation, early recognition of anatomic vascular anomalies can prevent vascular damage during harvesting and back-table surgery, and permits careful planning of reconstruction, when needed.

It is of extreme importance that organs for transplantation are retrieved without iatrogenic injury. Nijkamp et al. analyzed the incidence, risk factors, and clinical outcome of surgical injuries in 241 adult liver grafts [3]. The rate of hepatic artery injury was $12.5 \%$ and significantly higher in livers with replaced/accessory hepatic arteries. Post operative hepatic artery thrombosis was found in $23 \%$ of the patients who received a liver with arterial injury compared to $4 \%$ without $(\mathrm{P}<0.001)$. In a series of 4234 liver transplants the presence of a replaced/accessory hepatic artery predisposed to HAT which affected $5 \%$ of grafts [7]. In extreme cases an organ may be rendered untransplantable; $1 \%$ of kidney grafts are lost to iatrogenic injury [5]. Furthermore, unrecognised arterial injury prior to perfusing the liver with cold fluid would increase ischaemic injury.

In the presented cohort of 13 donors with CT images to review there was a single case of iatrogenic damage to the hepatic arterial anatomy. A RHA originating from the coeliac artery but passing posterior to the portal vein was divided. This demonstrates the importance of considering aberrant anatomy other than replaced or accessory he- patic arteries. The available imaging (Figure 1) demonstrates this anomaly but was not reviewed prior to organ retrieval highlighting the potential benefit of preoperative review. Whilst not asked directly to describe arterial anatomy adjacent to the portal vein 3 of 7 retrieval surgeons identified this abnormality.

By using co-incidental CT imaging the low incidence of cases for review and interpretation by the retrieving surgeon, not a radiologist, are two significant problems using the presented method. In a French study of donation after brain death donors a specific whole body CT angiogram was performed after declaring brain death [8]. Hepatic vascular abnormalities were observed in $8 / 27$ subjects. Though this technique also permits an evaluation of soft tissue abnormalities it requires performing an investigation for the purposes of donation and could be considered unethical, particularly so in cases of donation after cardiac death (DCD) where this would be impossible. DCD cases are technically more challenging as the entire retrieval process occurs with the donor in a pulseless state. It could be argued that the imaging would be better reviewed by a radiologist within the donor hospital though the high sensitivity, specificity, NPV and PPV of surgeons to correctly identify arterial anatomy is reassuring. It is an assumption of the authors that the availability of senior radiologists, typically late at night, to review these images in a timely manner would be limited. Either way, review of available imaging prior to organ retrieval would permit an evaluation of the hepatic vascular anatomy.

In summary the incidence of pre-mortem coincidental imaging of hepatic vascular anatomy is low. When present, however, retrieving surgeons can correctly identify arterial anatomy in the majority of occasions. Review of such imaging may therefore decrease the risk of iatrogenic damage during organ retrieval.

\section{REFERENCES}

[1] J. R. Hiatt, J. Gabbay and R. W. Busuttil, "Surgical Anatomy of the Hepatic Arteries in 1000 Cases," Annals of Surgery, Vol. 220, No. 1, 1994, pp. 50-52. doi:10.1097/00000658-199407000-00008

[2] L. M. Marin-Gomez, M. A. Gomez-Bravo, C. BernalBellido, J. M. Alamo-Martinez, G. Suarez-Artacho, et al., "Variability of the Extrahepatic Arterial Anatomy in 500 Hepatic Grafts,” Transplantation Proceedings, Vol. 42, No. 8, 2010, pp. 3159-3161. doi:10.1016/j.transproceed.2010.05.078

[3] D. M. Nijkamp, M. J. Slooff, C. S. van der Hilst, A. J. Ijtsma, K. P. de Jong, et al., "Surgical Injuries of Postmortem Donor Livers: Incidence and Impact on Outcome after Adult Liver Transplantation,” Liver Transplantation, Vol. 12, No. 9, 2006, pp. 1365-1370. doi:10.1002/lt.20809

[4] T. Soliman, F. Langer, H. Puhalla, H. Pokorny, T. Grun- 
berger, et al., "Parenchymal Liver Injury in Orthotopic Liver Transplantation,” Transplantation, Vol. 69, No. 10, 2000, pp. 2079-2084.

doi:10.1097/00007890-200005270-00018

[5] S. J. Wigmore, F. M. Seeney, H. C. Pleass, R. K. Praseedom and J. L. Forsythe, "Kidney Damage during Organ Retrieval: Data from UK National Transplant Database. Kidney Advisory Group,” Lancet, Vol. 354, No. 9185, 1999, pp. 1143-1146.

doi:10.1016/S0140-6736(98)09409-4

[6] N. A. Michels, "Newer Anatomy of the Liver and Its Variant Blood Supply and Collateral Circulation," The
American Journal of Surgery, Vol. 112, No. 3, 1966, pp. 337-347. doi:10.1016/0002-9610(66)90201-7

[7] J. P. Duffy, J. C. Hong, D. G. Farmer, R. M. Ghobrial, H. Yersiz, et al., "Vascular Complications of Orthotopic Liver Transplantation: Experience in More than $4200 \mathrm{~Pa}-$ tients," Journal of the American College Surgeons, Vol. 208, No. 5, 2009, pp. 896-903. doi:10.1016/j.jamcollsurg.2008.12.032

[8] A. Fregeville, C. De Bazelaire, A. Zagdanski, M. Albiter, F. Desgrandchamps, et al., "Value of Whole Body CTA in the Management of Brain-Dead Patients," Journal of Radiology, Vol. 91, No.1, 2010, pp. 37-44. 